\title{
Disability, Frailty and Depression in the community-dwelling older adults with Osteosarcopenia
}

\author{
Ki-Soo Park ${ }^{1,2}$, Gyeong-Ye Lee ${ }^{2}$, Young-Mi Seo ${ }^{2}$, Sung-Hyo Seo ${ }^{2,3}$ and Jun-II Yoo ${ }^{2,4^{*}}$
}

\begin{abstract}
Background: The purpose of this study was to investigate the prevalence of osteosarcopenia in the over 60-yearold community and to evaluate whether osteosarcopenia is associated with disability, frailty and depression.

Methods: This study was performed using the baseline data of Namgaram-2, among the 1010 surveyed subjects, 885 study subjects who were 60 years or older and had all necessary tests performed were selected. The KaigoYobo checklist (frailty), World Health Organization Disability Assessment Schedule (WHODAS) and Geriatric Depression Scale-Short Form-Korean (GDSSF-K) were used. The Asian Working Group for Sarcopenia (AWGS 2019) were applied in this study. Osteopenia was measured using data from dual energy X-ray absorptiometry (DEXA) and osteopenia was diagnosed when the T-score was less than - 1.0.

The study subjects were divided into four groups: the normal group, in which both sarcopenia and osteopenia were undiagnosed, osteopenia only, sarcopenia only and the osteosarcopenia group, which was diagnosed with both sarcopenia and osteopenia.
\end{abstract}

Results: Of the 885 subjects over 60 years old evaluated, the normal group comprised 34.0\%, the only osteopenia group $33.7 \%$, the only sarcopenia group $13.1 \%$, and the osteosarcopenia group 19.2\%. WHODAS (17.5, 95\% Cl: 14.820.1), Kaigo-Yobo (3.0, 95\% Cl: 2.6-3.4), and GDSSF mean score (4.6, 95\% Cl: 3.9-5.4) were statistically significantly higher in the osteosarcopenia group compared the other groups. Partial eta squared $\left(\eta_{p}{ }^{2}\right)$ of WHODAS $(0.199)$ and Kaigo-Yobo (0.148) values according to Osteosarcopenia were large, and GDSSF (0.096) was medium

Conclusions: Osteosarcopenia is a relatively common disease group in the older adults community that may cause deterioration of health outcomes. Therefore, when evaluating osteopenia or sarcopenia in the older adults, management of those in both disease groups should occur together.

Keywords: Disability, Frailty, Depression, Osteosarcopenia

\footnotetext{
* Correspondence: furim@daum.net

${ }^{2}$ Center for Farmer's Safety and Health, Gyeongsang National University Hospital, Jinju, Korea

${ }^{4}$ Department of Orthopaedic Surgery, Gyeongsang National University Hospital, 90 Chilamdong, Jinju, Republic of Korea

Full list of author information is available at the end of the article
}

(c) The Author(s). 2021 Open Access This article is licensed under a Creative Commons Attribution 4.0 International License, which permits use, sharing, adaptation, distribution and reproduction in any medium or format, as long as you give appropriate credit to the original author(s) and the source, provide a link to the Creative Commons licence, and indicate if changes were made. The images or other third party material in this article are included in the article's Creative Commons licence, unless indicated otherwise in a credit line to the material. If material is not included in the article's Creative Commons licence and your intended use is not permitted by statutory regulation or exceeds the permitted use, you will need to obtain permission directly from the copyright holder. To view a copy of this licence, visit http://creativecommons.org/licenses/by/4.0/ The Creative Commons Public Domain Dedication waiver (http://creativecommons.org/publicdomain/zero/1.0/) applies to the data made available in this article, unless otherwise stated in a credit line to the data. 


\section{Background}

As people live longer, the world's population is projected to age rapidly in most regions [1]. Korea became an aging society in 2018, and by 2026 Korea will become a super-aged society [2]. Older adults are increasing the prevalence of many chronic diseases, including osteoporosis and sarcopenia [3].

Although osteoporosis-based clinical outcomes and treatment recommendation studies have been increasingly popular in recent decades, sarcopenia and related studies have been less frequent [4]. In addition, sarcopenia has recently been classified as M62.84 in the ICD10-CM since 2016 [5].

Sarcopenia is most often seen in metabolic disorders such as diabetes mellitus, obesity, and other serious diseases, including congestive heart failure, chronic renal failure, and chronic obstructive pulmonary disease (COPD) [6-8]. Sarcopenia is substantially correlated with osteopenia (or osteoporosis) in older populations. Several studies have shown that sarcopenia and osteopenia (osteosarcopenia, OS) share similar risk factors and biological pathways [9]. OS is associated with severe physical impairment, which presents a major threat to the loss of freedom in later life. The combination of these two conditions exacerbates adverse health effects and creates a significant problem for the older adults. This combination has been presented as a "hazardous duet" that adds the predisposition of falling from sarcopenia to bone weakness in those with osteopenia [10].

In recent years, questionnaire research tools (World Health Disability Assessment Schedule (WHODAS), Kaigo-Yobo) have been developed to define disability and frailty as health outcomes and to measure these outcomes easily and effectively [11, 12]. OS patients have a higher risk of fracture from falling than those with osteopenia and sarcopenia alone. Studies have also shown that sarcopenia acts as a major risk factor for frailty [13]. However, a study on the relationships among OS, disability and frailty is lacking.

Therefore, the purpose of this study was to investigate the prevalence of OS in the over 60-year-old community and to evaluate whether OS is associated with disability, frailty and depression.

\section{Methods}

\section{Participants}

This study was performed using the baseline data of Namgaram-2, which was developed to study the relationship between the prevalence of musculoskeletal disorders and activity limitations in the older adults in 6 rural villages $[14,15]$.

At the time of the study, subjects who were diagnosed with chronic diseases and are currently receiving cancer treatment were excluded from the study. Only those with no cognitive problems were included in the study. The cognitive performance evaluation for participants with no cognitive function disorders was performed via an interview study utilizing the Korean version of the Mini-Mental State Examination for Dementia Screening (MMSE-DS) [16]. All surveys were evaluated by a medical professional.

After providing written informed consent, participants completed a questionnaire to assess cognitive function. Subsequently, fasting blood samples were collected from participants, followed by physical function evaluation. Among the 1,010 surveyed subjects, 885 were 60 years or older and had all necessary tests performed. These 885 population were selected as study subjects.

All investigations were conducted after obtaining participant consent and after being reviewed by the Institutional Review Board of our institution (approval number: GIRB-A16-0012).

\section{Materials}

Data on social demographic variables such as gender, age, marital status, economic level perceived by the person, and health behavior variable such as smoking was acquired. Also, survey results, such as one on whether or not the subject was affected by hypertension and diabetes, were used. In addition, the blood test measured hemoglobin, cholesterol, albumin, uric acid, $\gamma$-GTP, and creatinine.

\section{Frailty (Kaigo-Yobo checklist in Korean older adults)}

The Kaigo-Yobo checklist [17] was developed by researchers at the Tokyo Senior Research Institute, and the reliability and validity in Korean older adults have been confirmed [18]. The checklist consists of 15 questions and is a survey instrument that focuses on social activities and evaluation of daily life. The Kaigo-Yobo checklist is a complex domain phenotype aging assessment tool and comprises questions only. The checklist's 15 items consist of four products for nutritional status; three items for fall; two items for activities; two items for social relationships; and one item each for general health status, communication, mobility, and leisure activities. Furthermore, the appropriate answer to each query was 'Yes' or 'No,' and a score of one point was possible for each query. The final score is in the range of $0-15$ points, and the higher the score was indicated the higher the degree of frailty.

\section{WHODAS-12}

The World Health Organization (WHO) developed the WHO Disability Assessment Schedule (WHODAS) based on the International Classification of Functioning, Disability, and Health (ICF). WHODAS-12 measures the difficulties caused by health conditions by dividing the 
difficulties into six areas (cognition, mobility, self-care, getting along, life activities, and participation) [19].

All questions were measured with a 5-point Likert item system ( 1 point, not very difficult to 5 points, very severe). The final score is in the range of $0-100$ points, and the higher the score, the higher the degree of disability in everyday life.

\section{Depressive symptoms}

To understand the symptoms of depression in the older adults, the Geriatric Depression Scale-Short Form-Korean (GDSSF-K) adapted and developed for aged people in Korea was used [20]. The GDSSF-K has the advantage of comprising items that are easy for the older adults to understand relative to other current depression measurement tools. This scale of 15 items is graded from 0 to 15 points; higher values indicate worse depression.

\section{Osteosarcopenia}

Although several criteria have been proposed to define sarcopenia, the recent criteria of the Asian Working Group for Sarcopenia (AWGS 2019) were applied in this study [21].

Dual energy X-ray absorptiometry (DEXA) was used to measure muscle mass. In addition, the measured total appendicular skeletal muscle mass (ASM), excluding bone and fat, divided by the square of the height $\left(\mathrm{m}^{2}\right)$ was calculated $\left(\mathrm{ASM} / \mathrm{Ht}^{2}\right)$ and used as the skeletal muscle mass index (SMI). Sarcopenia was defined as SMI less than $7.0 \mathrm{~kg} / \mathrm{m}^{2}$ in men and less than $5.4 \mathrm{~kg} / \mathrm{m}^{2}$ in women. Muscle strength was evaluated by grip strength, and the measurement was conducted using the Smedley-type dynamometer (TKK 5401; Takei Scientific Instruments Co., Tokyo, Japan). Both hands were evaluated, twice each. Grip strength was used for analysis as one of the four measured values. The maximum value was used as a reference level, for men below $28 \mathrm{~kg}$ and for women below $18 \mathrm{~kg}$.

Osteopenia was measured using data from dual energy $\mathrm{X}$-ray absorptiometry (DEXA) and osteopenia was diagnosed when the T-score was less than - 1.0 [22].

The study subjects were divided into four groups [23]: the normal group, in which both sarcopenia and osteopenia were undiagnosed, and the osteosarcopenia group, which was subdivided into those diagnosed with both sarcopenia and osteopenia, osteopenia only, and sarcopenia only.

\section{Statistical analysis}

The general characteristics of the participants generated descriptive statistics; the chi-square test was conducted on categorical variables, and the ANOVA test was performed on continuous variables. Post-hoc analysis was performed using the Tukey method. The odds ratios
(OR) of osteosarcopenia prevalence according to socioeconomic status were analyzed by logistic regression.

The comparison of WHODAS, Kaigo-Yobo, and GDSSF values among the four groups of study subjects occurred after adjustment for gender, age, marital status, economic level, smoking status, hypertension, diabetes, hemoglobin, uric acid, albumin, r-GTP, creatinine, and cholesterol [24-26]. Adjusted means and their $95 \%$ confidence intervals (CIs) were estimated by general linear model (GLM) and the post-hoc test is based on Turkey method. And Partial eta squared $\left(\eta_{\mathrm{p}}{ }^{2}\right)$ was calculated to determine the effect size, using the $0.0099,0.0588$, and 0.1379 considered as small, medium, and large effect sizes [27]. The SAS Version 9.4 program (SAS Institute Inc., Cary, NC) was used as the analysis tool, and the significance level was set to 0.05 .

\section{Result}

\section{General characteristics}

Of the 885 older adults people over 60 years old evaluated, 594 (67.1\%) were women. The average participant age was $70.3 \pm 6.2$ years, $561(63.4 \%)$ participants had spouses, and 577 (69.7\%) had a higher than average economic status. The smoking rate was $7.5 \%, 447$ (50.5\%) were diagnosed with hypertension, and 192 (21.7\%) were diagnosed with diabetes.

The diagnosis of osteopenia and osteoporosis (below T-score - 1.0) occurred in 468 subjects $(47.1 \%)$, and sarcopenia was diagnosed in $286(32.3 \%)$ (Table 1$)$.

\section{Osteosarcopenia according to general characteristics}

The normal group comprised 34\% (301/885), the only osteopenia group $33.7 \%(298 / 885)$, the only sarcopenia

Table 1 General characteristics (Total number $=885$ )

\begin{tabular}{llll}
\hline Variables & & Number & $\%$ \\
\hline Sex & Male & 291 & $32.9 \%$ \\
Age (years) & Female & 594 & $67.1 \%$ \\
Spouse & mean \pm SD & $70.3 \pm 6.2$ & \\
Economic status & Yes & 561 & $63.4 \%$ \\
& No & 324 & $36.6 \%$ \\
& Low & 268 & $30.3 \%$ \\
Smoking & Middle & 465 & $52.5 \%$ \\
& High & 152 & $17.2 \%$ \\
Hypertension & Non-smoker & 819 & $92.5 \%$ \\
& Smoker & 66 & $7.5 \%$ \\
Diabetes mellitus & No & 438 & $49.5 \%$ \\
& Yes & 447 & $50.5 \%$ \\
& No & 693 & $78.3 \%$ \\
\hline
\end{tabular}


Table 2 Osteosarcopenia prevalence according to general characteristics

\begin{tabular}{|c|c|c|c|c|c|c|c|c|c|c|}
\hline \multirow[t]{2}{*}{ Variables } & & \multicolumn{2}{|c|}{ Normal } & \multicolumn{2}{|c|}{ Only Osteopenia } & \multicolumn{2}{|c|}{ Only Sarcopenia } & \multicolumn{2}{|c|}{ Osteo-sarcopenia } & \multirow{2}{*}{$\begin{array}{l}p \text { - } \\
\text { value }\end{array}$} \\
\hline & & $\mathrm{N}$ & $(\%)$ & $\mathbf{N}$ & (\%) & $\mathbf{N}$ & $(\%)$ & $\mathrm{N}$ & (\%) & \\
\hline Total & & 301 & 34.0 & 298 & 33.7 & 116 & 13.1 & 170 & 19.2 & \\
\hline \multirow[t]{2}{*}{ Sex } & Male & 172 & 57.1 & 75 & 25.2 & 26 & 22.4 & 18 & 10.6 & \multirow[t]{2}{*}{$<0.001$} \\
\hline & Female & 129 & 42.9 & 223 & 74.8 & 90 & 77.6 & 152 & 89.4 & \\
\hline \multicolumn{2}{|l|}{ OR (female/male) } & \multicolumn{2}{|c|}{$\mathrm{OR}=1$} & \multicolumn{2}{|c|}{$\mathrm{OR}=4.0(p<0.001)$} & \multicolumn{2}{|c|}{$\mathrm{OR}=4.6(p<0.001)$} & \multicolumn{2}{|c|}{$\mathrm{OR}=11.3(p<0.001)$} & \\
\hline Age (years) & mean $\pm \mathrm{SD}$ & $68.2 \pm$ & & $68.8=$ & & $73.1=$ & & $75.0 \pm$ & & $<0.001$ \\
\hline \multicolumn{2}{|l|}{ OR (unit: years) } & \multicolumn{2}{|c|}{$\mathrm{OR}=1$} & \multicolumn{2}{|c|}{$\mathrm{OR}=1.0(p=0.192)$} & \multicolumn{2}{|c|}{$\mathrm{OR}=1.2(p<0.001)$} & \multicolumn{2}{|c|}{$\mathrm{OR}=1.2(p<0.001)$} & \\
\hline \multirow[t]{2}{*}{ Spouse } & Yes & 241 & 80.1 & 193 & 64.8 & 54 & 46.6 & 73 & 42.9 & \multirow[t]{2}{*}{$<0.001$} \\
\hline & No & 60 & 19.9 & 105 & 35.2 & 62 & 53.4 & 97 & 57.1 & \\
\hline \multicolumn{2}{|l|}{ OR (no/yes) } & \multicolumn{2}{|c|}{$\mathrm{OR}=1$} & \multicolumn{2}{|c|}{$\mathrm{OR}=2.2(p<0.001)$} & \multicolumn{2}{|c|}{$\mathrm{OR}=4.6(p<0.001)$} & \multicolumn{2}{|c|}{$\mathrm{OR}=5.3(p<0.001)$} & \\
\hline \multirow[t]{3}{*}{ Economic status } & Low & 76 & 25.3 & 87 & 29.2 & 45 & 38.8 & 60 & 35.3 & \multirow[t]{3}{*}{0.002} \\
\hline & Middle & 153 & 50.8 & 168 & 56.4 & 56 & 48.3 & 88 & 51.8 & \\
\hline & High & 72 & 23.9 & 43 & 14.4 & 15 & 12.9 & 22 & 12.9 & \\
\hline \multicolumn{2}{|l|}{ OR (middle/low) } & \multicolumn{2}{|c|}{$\mathrm{OR}=1$} & \multicolumn{2}{|c|}{$\mathrm{OR}=1.0(p=0.090)$} & \multicolumn{2}{|c|}{$\mathrm{OR}=0.6(p=0.858)$} & \multicolumn{2}{|c|}{$\mathrm{OR}=0.7(p=0.431)$} & \\
\hline \multicolumn{2}{|l|}{ OR (high/low) } & $\mathrm{OR}=1$ & & $\mathrm{OR}=$ & .004) & $\mathrm{OR}=$ & .009) & $\mathrm{OR}=\mathrm{C}$ & 003) & \\
\hline Smoking & No & 272 & 90.4 & 286 & 96.0 & 114 & 98.3 & 166 & 97.6 & $<0.001$ \\
\hline & Yes & 29 & 9.6 & 12 & 4.0 & 2 & 1.7 & 4 & 2.4 & \\
\hline OR (yes/no) & & $\mathrm{OR}=1$ & & $O R=$ & .588) & $\mathrm{OR}=$ & .053) & $\mathrm{OR}=\mathrm{C}$ & 194) & \\
\hline Hypertension & No & 141 & 46.8 & 178 & 59.7 & 46 & 39.7 & 73 & 42.9 & $<0.001$ \\
\hline & Yes & 160 & 53.2 & 120 & 40.3 & 70 & 60.3 & 97 & 57.1 & \\
\hline OR (yes/no) & & $\mathrm{OR}=1$ & & $\mathrm{OR}=$ & $.160)$ & $\mathrm{OR}=$ & $.187)$ & $\mathrm{OR}=1$ & 414) & \\
\hline
\end{tabular}

group $13.1 \%(116 / 885)$, and the OS group $19.2 \%(116 /$ 885) (Table 2).

The distribution of osteosarcopenia by male and female was significantly different $(p<0.001)$, and compared to the normal group, the ORs of the other 3 groups and sex were significantly associated with 4.0 $(p<0.001), 4.6(p<0.001), 11.3(p<0.001)$, respectively. and age was statistically higher in the OS group (75 \pm 5.8 years, $P<0.001)$ compared to other groups. There was also a statistical difference by spouse's presence $(p<$ 0.001 ) and at the economic level, the low group had a significant difference of osetosarcoipenia (22.4\%), while the group with a high economic level (14.5\%) ( $p=$ $0.002)$. The distribution of osteosarcopenia was also significantly different depending on whether hypertension and diabetes were present $(p<0.001)$.

WHODAS, Kaigo-Yobo, GDSSF scores according to the presence of osteosarcopenia after adjusting covariates WHODAS disability mean scores were normal (8.6, $95 \%$ CI: 6.3-11.0), only osteopenia (9.4, $95 \% \mathrm{CI}$ : 7.1-11.8), only sarcopenia (16.4, $95 \% \mathrm{CI}$ : 13.5-19.3), and OS (17.5, $95 \%$ CI: 14.8-20.1), the OS group had statistically significantly higher WHODAS scores compared to the other groups $(P<0.001)$, and the effect size (partial eta squared $\left(\eta_{\mathrm{p}}{ }^{2}\right)$ ) of the WHODAS mean according to the four groups was 0.199 , which was large.

Kaigo-Yobo frailty mean scores were normal (1.6, $95 \%$ CI: 1.2-2.0), only osteopenia (1.9, $95 \%$ CI: 1.5-2.2), only sarcopenia $(2.7,95 \% \mathrm{CI}: 2.3-3.2)$, and OS (3.0, $95 \% \mathrm{CI}$ : 2.6-3.4), the OS group had statistically significantly higher depressive scores compared to the other groups $(P<0.001)$, and the effect size of the frailty means according to the four groups was 0.148 , which was large.

The GDSSF depression mean scores were normal (2.7, $95 \%$ CI: $2.1-3.4)$, only osteopenia (3.1, 95 \% CI: $2.4-3.7)$, only sarcopenia (4.3, $95 \%$ CI: 3.5-5.1), and OS (4.6, $95 \%$ CI: 3.9-5.4), the OS group had statistically significantly higher depressive scores compared to the other groups $(P<0.001)$, and the effect size of the depressive means according to the four groups was 0.096, which was medium (Table 3 ).

\section{Osteosarcopenia according to the presence of frailty}

The prevalence of OS (37.7\%) in the frailty group was statistically significantly higher than that of OS (14.9\%) in the robust group. $(P<0.001)$ (Fig. 1) And, compared to the robust group, the OR between the normal group 
Table 3 WHODAS, Kaigo-Yobo, GDSSF score according to the presence of osteosarcopenia

\begin{tabular}{|c|c|c|c|c|c|c|c|c|c|c|c|c|c|c|}
\hline & \multicolumn{3}{|c|}{ Normal } & \multicolumn{3}{|c|}{ Only Osteopenia } & \multicolumn{3}{|c|}{ Only Sarcopenia } & \multicolumn{3}{|c|}{ Osteosarcopenia } & \multirow{2}{*}{$\begin{array}{l}\text { Effect size } \\
\text { Partial eta squared }\end{array}$} & \multirow{2}{*}{$\begin{array}{l}p \\
\text { value }\end{array}$} \\
\hline & mean & $\mathrm{SE}$ & $95 \% \mathrm{Cl}$ & mean & SE & $95 \% \mathrm{Cl}$ & mean & SE & $95 \% \mathrm{Cl}$ & mean & SE & $95 \% \mathrm{Cl}$ & & \\
\hline WHODAS & 8.6 & 1.20 & $6.3-11.0$ & 9.4 & 1.20 & $7.1-11.8$ & 16.4 & 1.48 & $13.5-19.3$ & 17.5 & 1.35 & $14.8-20.1$ & 0.199 & $<0.0$ \\
\hline aKaigo-Yobo & 1.6 & 0.18 & $1.2-2.0$ & 1.9 & 0.18 & $1.5-2.2$ & 2.7 & 0.23 & $2.3-3.2$ & 3.0 & 0.21 & $2.6-3.4$ & 0.148 & $<0$ \\
\hline${ }^{\mathrm{a}} \mathrm{GDSSF}$ & 2.7 & 0.34 & $2.1-3.4$ & 3.1 & 0.34 & $2.4-3.7$ & 4.3 & 0.42 & $3.5-5.1$ & 4.6 & 0.38 & $3.9-5.4$ & 0.096 & $<0.0$ \\
\hline
\end{tabular}

${ }^{a}$ Adjusting factors: Age, Sex, Spouse, Economic status, Smoking, Hypertension, Diabetes mellitus, Osteopenia, Sarcopenia, Hb, Uric acid, Albumin, $\gamma$-GTP, Creatine, Cholesterol

WHODAS World Health Organization Disability Assessment Schedule, GDSSF Geriatric Depression Scale-Short Form

and only osteopenia was $2.97(p<0.001)$ in the frailty group, the OR with only sarcopenia was $9.48(p<0.001)$ and the OR with the osteosarcopenia group was 9.84 $(p<0.001)$.

\section{Discussion}

The main findings of this study were: (1) the prevalence of OS in the community using the new AWGS 2019 criteria was $19.2 \%$; (2) the prevalence of OS was significantly higher in older women; (3) $\mathrm{Hb}$ and albumin levels were lower in subjects with OS; and (4) WHODAS, Kaigo-Yobo, and GDSSF scores were all higher in the OS group after adjusting for general characteristics that were different among the four groups. Therefore, we suggest that osteosarcopenia exacerbates frailty, disability and depression, thereby increasing susceptibility to various chronic diseases.
Huo et al. [23] conducted a cross-sectional study of 680 community-dwelling older individuals and reported that the percentage with OS was almost $40 \%$. A study of community-dwelling Chinese elders (older than age 65) found the prevalence of OS to be $10.4 \%$ in men and $15.1 \%$ in women. In this study, we observed a prevalence similar to that of community-dwelling Chinese elders. Although the prevalence of OS varies greatly depending on the criteria for diagnosis of osteopenia or sarcopenia, OS is not uncommon in older adults women in the community. In particular, considering the commonality of concomitant osteopenia (including osteoporosis) and sarcopenia in the community, DEXA analyses should measure not only bone density but also muscle mass.

Our results suggest that, in older adults women suspected of being malnourished (low $\mathrm{Hb}$, albumin levels), there may be a risk of impairment due to reduced

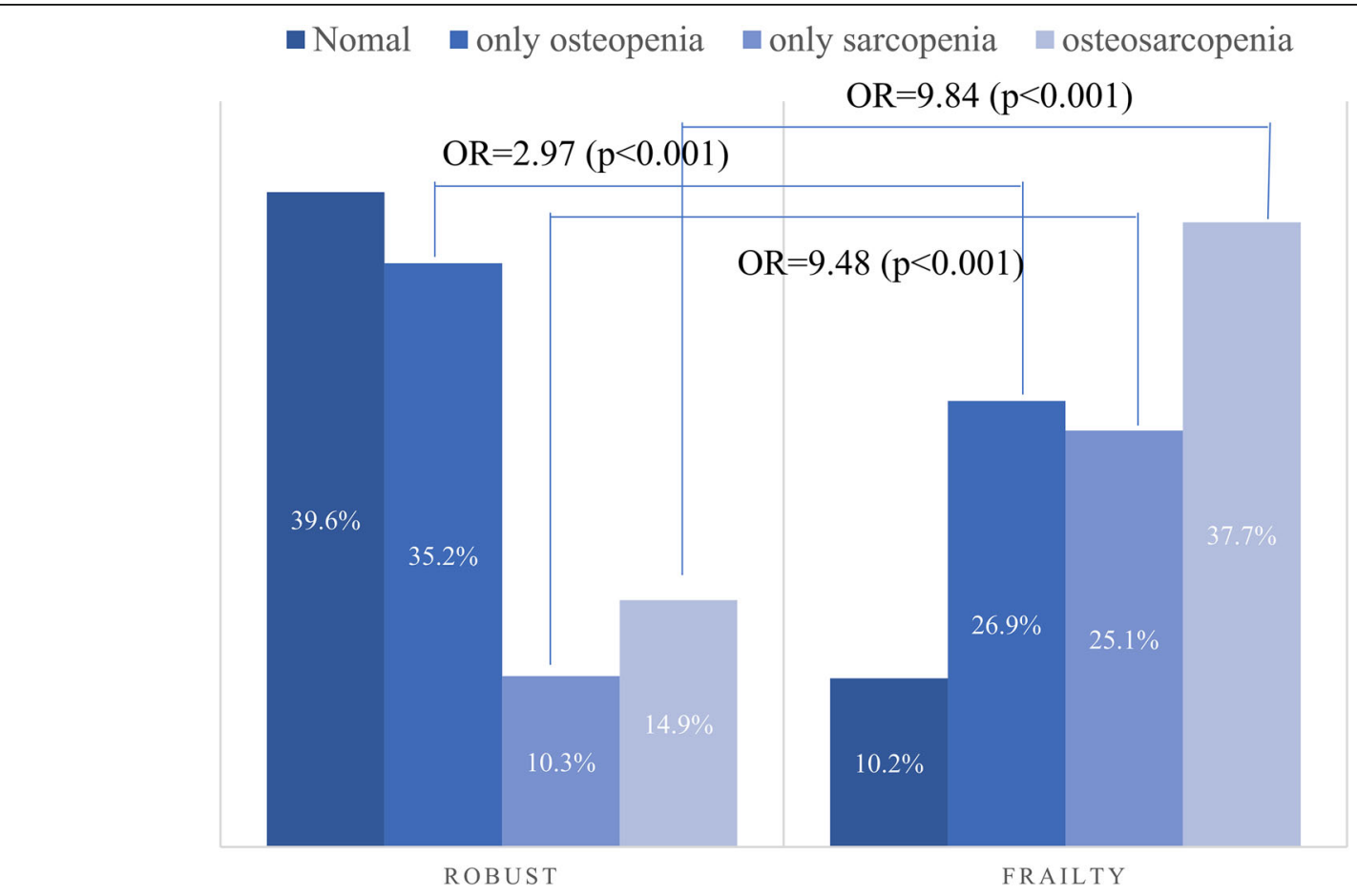

Fig. 1 Osteosarcopenia according to the presence of frailty- 
muscle mass and strength and decreased bone density. There is also a high risk of disability and frailty in these subjects.

WHODAS disability scores were significantly higher in the only osteopenia, only sarcopenia, and OS groups compared to the normal group. Frailty and depression scores were significantly higher in the only sarcopenia and OS groups than in the normal and osteopenia alone groups. Therefore, BMD test performance should be accompanied by the sarcopenia test.

For OS, fall, fracture and mortality rates have been reported to be higher than those with bone loss only or muscle loss only, but other studies have reported different results $[28,29]$. However, there are no studies reporting on associations with disability and frailty among these groups. Compared to the osteopenia alone group, the WHODAS score showed a clinically significant difference of 8.8 points when sarcopenia was present suggesting that depression is more likely to impact people with sarcopenia than osteopenia. This is consistent with the results of previous research that identified a correlation between disability, frailty, and depression and sarcopenia. While osteopenia was assessed only by bone density testing, sarcopenia was diagnosed as having a decrease in muscle mass as well as a decrease in muscle strength (function), which may be lower in disability, frailty, and depression. In other words, osteopenia may not have progressed to the point of requiring treatment for assistance in daily life activities. However, sarcopenia may be associated with more activity limitation than osteopenia (not osteoporosis).

This study has several limitations. First, the design was cross-sectional. Therefore, causative relationships could not be ascertained. Second, Because the study subjects are not representative samples, caution should be used when expanding the results of the study.

Nevertheless, our research has strength. The study is meaningful as the first to analyze the relationship between OS and disability, depression, and frailty. Future research should not only focus on OS diagnosis and clinical outcomes such as falls, fractures, and death, but also on the OS patient's quality of life and the occurrence of disability.

\section{Conclusions}

OS is a relatively common disease in the community and may cause deterioration of health outcomes. Therefore, concurrent evaluation and management of osteopenia and sarcopenia in the older adults is required.

\section{Abbreviations}

WHODAS: WHO Disability Assessment Schedule; GDSSF-K: Geriatric

Depression Scale-Short Form-Korean; ICD-10-CM: International Classification of Disease, Tenth Revision, Clinical Modification; COPD: Chronic obstructive pulmonary disease; OS: Osteosarcopenia; MMSE-DS: Mini-Mental State
Examination for Dementia Screening; ICF: International Classification of Functionin; AWGS: Asian Working Group for Sarcopenia; DEXA: Dual energy X-ray absorptiometry; ASM: Appendicular skeletal muscle mass; SMI: Skeletal muscle mass index

\section{Acknowledgements}

This work was supported by the centers for farmer's safety and health, ministry of agriculture, food, and rural affairs.

\section{Authors' contributions}

All authors read and approved the manuscript. Conceived and designed the experiments: KSP and JIY. Analyzed the data: GYL, YMS, Contributed reagents/materials/analysis tools: SHS. Wrote the paper: KSP and JIY.

\section{Funding}

The authors have no support or funding to report.

\section{Availability of data and materials}

The datasets used and/or analysed during the current study available from the corresponding author on reasonable request.

\section{Ethics approval and consent to participate}

All investigations were conducted after obtaining consent which obtained from study participants was written and after being reviewed by the Institutional Review Board of Gyeongsang national university hospital (approval number: GIRB-A16-0012).

Participants provided consent for use of data in research projects. The data are centrally housed at GNUH. The data were accessed after an analysis plan for this manuscript was approved by the GIRB Publications Committee.

Consent for publication

Not applicable.

\section{Competing interests}

All authors have no conflict of interest.

\section{Author details}

'Department of Preventive Medicine, Institute of Health Sciences, College of Medicine, Gyeongsang National University, Jinju, Korea. ${ }^{2}$ Center for Farmer's Safety and Health, Gyeongsang National University Hospital, Jinju, Korea. ${ }^{3}$ Department of Information \& Statistics, College of Natural Science, Gyeongsang National University, Jinju, Korea. ${ }^{4}$ Department of Orthopaedic Surgery, Gyeongsang National University Hospital, 90 Chilamdong, Jinju, Republic of Korea.

Received: 9 May 2020 Accepted: 11 January 2021

Published online: 19 January 2021

\section{References}

1. United Nations. Department of Economic and Social Affairs, Population Division. World population ageing, 2019 highlights. 2020.

2. Kim KJ, Shin J, Choi J, Won CW. Discrepancies in the Prevalence of Known Frailty Scales: Korean Frailty and Aging Cohort Study. Ann Geriatr Med Res. 2018:22:137-44.

3. Curtis E, Litwic A, Cooper C, Dennison E. Determinants of muscle and bone aging. J Cell Physiol. 2015;230:2618-25.

4. Han A, Bokshan SL, Marcaccio SE, DePasse JM, Daniels AH. Diagnostic Criteria and Clinical Outcomes in Sarcopenia Research: A Literature Review. J Clin Med. 2018;7. doi:https://doi.org/10.3390/jcm7040070.

5. Anker SD, Morley JE, von Haehling S. Welcome to the ICD-10 code for sarcopenia. J Cachexia Sarcopenia Muscle. 2016;7:512-4.

6. von Haehling S. Muscle wasting and sarcopenia in heart failure: a brief overview of the current literature. ESC Heart Fail. 2018:5:1074-82.

7. Lang T, Streeper T, Cawthon P, Baldwin K, Taaffe DR, Harris TB. Sarcopenia: etiology, clinical consequences, intervention, and assessment. Osteoporos Int. 2010;21:543-59.

8. Kim SH, Shin MJ, Shin YB, Kim KU. Sarcopenia Associated with Chronic Obstructive Pulmonary Disease. J Bone Metab. 2019;26:65-74.

9. Kirk B, Zanker J, Duque G. Osteosarcopenia: epidemiology, diagnosis, and treatment-facts and numbers. J Cachexia Sarcopenia Muscle. 2020. 
10. Yoo J-I, Ha Y-C. Review of Epidemiology, Diagnosis, and Treatment of Osteosarcopenia in Korea. J Bone Metab. 2018;25:1.

11. Ferrer MLP, Perracini MR, Rebustini F, Buchalla CM. WHODAS 2.0-BO: normative data for the assessment of disability in older adults. Rev Saude Publica. 2019;53:19.

12. Kojima G, Taniguchi Y, Kitamura A, Shinkai S. Are the Kihon Checklist and the Kaigo-Yobo Checklist Compatible With the Frailty Index? J Am Med Dir Assoc. 2018;19:797-800.e2.

13. Greco EA, Pietschmann P, Migliaccio S. Osteoporosis and Sarcopenia Increase Frailty Syndrome in the Elderly. Front Endocrinol (Lausanne). 2019; 10. doi:https://doi.org/10.3389/fendo.2019.00255.

14. Kim M-J, Kang B-H, Park S-H, Kim B, Lee G-Y, Seo Y-M, et al. Association of the Western Ontario and McMaster Universities Osteoarthritis Index (WOMAC) with Muscle Strength in Community-Dwelling Elderly with Knee Osteoarthritis. Int J Environ Res Public Health. 2020;17.

15. Kim M, Yoo JI, Kim MJ, Na JB, Lee SI, Park KS. Prevalence of Upper Extremity Musculoskeletal Diseases and Disability among Fruit Tree Farmers in Korea: Cross-Sectional Study. Yonsei Med J. 2019;60:870-5.

16. Kim TH, Jhoo JH, Park JH, Kim JL, Ryu SH, Moon SW, et al. Korean version of mini mental status examination for dementia screening and its' short form. Psychiatry Investig. 2010;7:102-8.

17. Shinkai S, Watanabe N, Yoshida H, Fujiwara Y, Amano H, Lee S, et al. [Research on screening for frailty: development of "the Kaigo-Yobo Checklist"]. Nihon Koshu Eisei Zasshi. 2010;57:345-54.

18. Hwang HS, Yoon JL, Park BJ, Choi HR, Kwon IS, Shinkai S, et al. The Validity and Reliability of the Kaigo-Yobo Checklist in Korean Elderly. J Korean Geriatr Soc. 2012;16:121.

19. Song JM, Lee HJ. Korean Cultural Adaptation of WHODAS 2.0 (36-Item Version): Reliability and Linking to ICF. J Kor Phys Ther. 2018;30:246-55

20. Kim JY, Park JH, Lee JJ, Huh Y, Lee SB, Han SK, et al. Standardization of the Korean Version of the Geriatric Depression Scale: Reliability, Validity, and Factor Structure. Psychiatry Investig. 2008;5:232-8.

21. Chen L-K, Woo J, Assantachai P, Auyeung T-W, Chou M-Y, lijima K, et al. Asian Working Group for Sarcopenia: 2019 Consensus Update on Sarcopenia Diagnosis and Treatment. J Am Med Dir Assoc. 2020;21:300-7.e2

22. Kanis JA, Burlet N, Cooper C, Delmas PD, Reginster J-Y, Borgstrom F, et al. European guidance for the diagnosis and management of osteoporosis in postmenopausal women. Osteoporos Int. 2008;19:399-428.

23. Huo YR, Suriyaarachchi P, Gomez F, Curcio CL, Boersma D, Muir SW, et al. Phenotype of osteosarcopenia in older individuals with a history of falling. J Am Med Dir Assoc. 2015;16:290-5.

24. Wang Y-J, Wang Y, Zhan J-K, Tang Z-Y, He J-Y, Tan P, et al. SarcoOsteoporosis: Prevalence and Association with Frailty in Chinese Community-Dwelling Older Adults. International Journal of Endocrinology. 2015;2015:1-8.

25. Almeida OP, Flicker L, Rees M. Depression, dementia and cognition in older people. Maturitas. 2014;79:133-5.

26. Kirk B, Zanker J, Duque G. Osteosarcopenia: epidemiology, diagnosis, and treatment-facts and numbers. Journal of Cachexia Sarcopenia Muscle. 2020;11:609-18

27. Richardson JT. Eta squared and partial eta squared as measures of effect size in educational research. Educ Res Rev. 2011;6:135-47.

28. Scott D, Seibel M, Cumming R, Naganathan V, Blyth F, Le Couteur DG, et al. Does Combined Osteopenia/Osteoporosis and Sarcopenia Confer Greater Risk of Falls and Fracture Than Either Condition Alone in Older Men? The Concord Health and Ageing in Men Project. J Gerontol A Biol Sci Med Sci. 2019;74:827-34

29. Balogun S, Winzenberg T, Wills K, Scott D, Callisaya M, Cicuttini F, et al. Prospective associations of osteosarcopenia and osteodynapenia with incident fracture and mortality over 10 years in community-dwelling older adults. Arch Gerontol Geriatr. 2019:82:67-73.

\section{Publisher's Note}

Springer Nature remains neutral with regard to jurisdictional claims in published maps and institutional affiliations.

Ready to submit your research? Choose BMC and benefit from:

- fast, convenient online submission

- thorough peer review by experienced researchers in your field

- rapid publication on acceptance

- support for research data, including large and complex data types

- gold Open Access which fosters wider collaboration and increased citations

- maximum visibility for your research: over $100 \mathrm{M}$ website views per year

At $\mathrm{BMC}$, research is always in progress.

Learn more biomedcentral.com/submissions 